\title{
User Experiences and Expectations of Vibrotactile, Thermal and Squeeze Feedback in Interpersonal Communication
}

\author{
Katja Suhonen \\ Tampere University of Technology, \\ Human-Centered Technology, \\ P.O.Box 589, 33101 Tampere, \\ Finland \\ katja.suhonen@tut.fi
}

\author{
Kaisa Väänänen-Vainio-Mattila \\ Tampere University of Technology, \\ Human-Centered Technology, \\ P.O.Box 589, 33101 Tampere, \\ Finland \\ kaisa.vaananen-vainio- \\ mattila@tut.fi
}

\author{
Kalle Mäkelä \\ Tampere University of Technology, \\ Department of Electronics, \\ P.O.Box 692, 33101 Tampere, \\ Finland \\ kalle.makela@tut.fi
}

\begin{abstract}
The haptic modality provides a new channel for interpersonal communication through technology by utilizing the sense of touch. In the development of novel haptic communication devices, it is essential to explore the potential users' perceptions of such a communication channel. To this end, we conducted two explorative user studies with two early prototypes that demonstrated three different haptic feedback types: vibrotactile, thermal and squeeze feedback. We arranged focus groups and interviews to study the participants' experiences, expectations and ideas of using these haptic technologies in interpersonal communication. The findings show, for example, that people prefer to use haptic communication mainly with people close to them. Haptics can be used for pragmatic purposes as well as in emotional communication, for example in mimicking touch between the communication partners. Squeezes were experienced as the most pleasant type of haptic feedback. Furthermore, the participants preferred receiving haptic stimuli to their hand area, through a mobile phone or a wristband-like device. We argue that using early prototypes in an early stage of research process in focus groups and interviews is especially useful for stimulating idea generation and discussions about expectations and experiences of haptic technologies.
\end{abstract}

Haptic interaction, user experience, user expectations, vibrotactile, thermal, and pressure stimulus.

\section{INTRODUCTION}

Interpersonal communication has been mediated with technology mainly through speech, text, multimedia messages over email and mobile phone, and videoconferencing. Even though the sense of touch is essential for human communication, it has received less attention in the development of human-technology interaction. Haptic user interfaces have been explored in technology prototypes that use various forms of feedback based on the sense of touch (see e.g. Bauman et al. 2010; Heikkinen et al. 2009b; Wilson et al. 2011) and some initial studies of the acceptability of haptic interaction on mobile devices have been conducted, for example by Heikkinen et al. (2009a). However, studies of user experience of the various forms of haptic interaction are still scarce.

We have been investigating user experience of various forms of human interaction, especially in mobile communication. Our intention is to shed light on both pragmatic (i.e. utility-oriented) and hedonic (i.e. pleasurable, non-goal-oriented) motivations of users (see e.g. Hassenzahl, 2004) in such interaction applications. Our research has explored various modalities and their combinations through user needs studies, prototyping and user evaluations. In our user experience research, we have been applying different methods, for example focus groups, for involving users into the early phases of research and development processes. Exploring users' expectations of novel technologies and their ideas for the design of applications in an early stage can provide valuable information that can be used to design for better user experience. We believe this approach to be essential for human-centred development of new communication technologies.

The goal of the present research - which is a combination of two user studies - was to explore people's experiences and expectations of using haptic feedback in interpersonal communication. The aim was to understand how, in what kind of situations, and for what kind of communication purposes haptic feedback could be used. The first 
study focused on thermal and vibrotactile feedback, which were studied in four focus groups. The second study consisted of six pair interviews focusing on squeeze feedback. The studies had similar research questions, only the studied forms of haptic feedback were different. Both studies were explorative and qualitative and utilized prototypes as stimulus material to gain more realistic user feedback. The studies provided information of user perceptions, expectations and ideas for potential uses of haptics in communication, which can be used in interaction studies and in the development of new haptic prototypes.

The next section presents related work on haptic interaction in interpersonal communication. Sections 3 and 4 introduce the two user studies. Section 5 presents the results of both studies, and Section 6 discusses the results and methods that were used, and concludes the paper.

\section{RELATED WORK}

Haptic feedback provides a channel to convey technology-mediated information directly to the user's skin. Perception of haptic stimuli comprises the kinaesthetic system, which relates to movement and position of limbs, and the tactile system, which refers to stimulation from various receptor cells in our skin (Haans and ljsselstein, 2006). Through the sense of touch we can detect different types of haptic stimuli, such as vibration, temperature, pressure, pain, and position (Haans and ljsselstein, 2006). In our studies, we have focused on the first three - vibration, temperature and pressure. They are natural means of human interaction that can also be used mediated via technology. Vibration reminds the user of fingers tickling the skin, and a feeling of pressure can be associated to grip of a hand or a hug, whereas warmth is an indication of body heat.

Haptic interaction has been studied in different contexts. For instance, communicating social presence has been studied with prototypes such as the Huggy Pajama, a system that enables parents and children to hug one another through a hugging interface device and a wearable, hug reproducing pajama (Teh et al. 2008); the SqueezeMe, a hugging vest for therapeutic purposes that has inflatable pneumatic chambers (Vaucelle et al. 2009); and the Thermal Hug Belt, which also conveys hugs, but with warmth instead of pressure (Gooch and Watts, 2010). Other examples of mediating human touch with technology include a wristband with tapping and squeezing mechanisms to emulate human attention-getting practices (Baumann et al. 2010) and an armband for squeezing or holding the upper arm with an aim to convey emotions (Wang and Quek, 2010). Most of the haptic prototypes in literature are wearable or hand-held and usually also mobile. An example of haptics for mobile phones is ComTouch by Chang et al. (2002), which is a device that is fitted over a traditional mobile phone and translates finger pressure to vibrotactile stimuli. In their study, users found the haptic channel could be used for emphasis, turn-taking, mimicry and encoding of specific meanings.

Studies of the interaction between the skin and haptic devices have concerned, for example, the detection of and changes in thermal stimuli in static (Salminen et al. 2011; Wettach et al. 2007; Wilson et al. 2011) and mobile contexts (Wilson et al. 2011). The ability of thermal stimuli to convey usable pragmatic information to the user has been studied for example in a navigation scenario by Wettach et al. (2007). Heikkinen et al. have explored potential uses of gestures in haptic communication (2009b) and users' ability to interpret the meaning of vibrotactile messages (2011).

These examples present only a glimpse of the literature on haptic interaction research and more can be found, for example, in a review by Haans and IJsselstein (2006). However, there seem to be fewer studies regarding the users' point of view in the early phase of ideating potential use scenarios and what they feel is important in haptic interaction. Examples of the few exceptions include a study by Heikkinen et al. (2009a) about what people expect from haptic communication with mobile devices, the research by Lee and Lim (2010) on how heat can be used for mediating valuable interpersonal communication, and a series of studies by Mueller et al. (2005) in which they applied participatory design to study remote intimacy. Mueller et al. examined personal interactions of intimate couples with focus groups and cultural probes, and built a prototype to give hugs remotely, and finally used the prototype in a workshop to generate new ideas. They found that using a prototype helped the users better understand the aim of the research and why their participation was needed, making it a more enjoyable experience for everybody. In our study, we gave the users an opportunity to test two prototypes and to share their perceptions of using these haptic technologies in interpersonal communication.

\section{USER STUDY OF THERMAL AND VIBROTACTILE FEEDBACK}

The first study with four focus group sessions aimed at having the users collaboratively ideate scenarios for using thermal and vibrotactile feedback in interpersonal communication and 
exploring their perceptions of the topic, with the help of a haptic prototype. In particular, we wanted to explore in what kind of situations, for what kind of communication purposes and in what ways people would prefer to use haptics. The emphasis was on thermal feedback, as it is a newer way of using haptics in user interfaces, although vibration was also studied.

\subsection{The prototype with thermal and vibrotactile feedback}

The prototype consisted of an elastic band with a main module, a thermal actuator module (a Peltier element) and a vibrating motor actuator module, which were connected through an interface box to a computer with serial output (see Figure 1). The band was originally designed to be worn around the head, but by using different attachment styles it can be mounted also on other body locations, such as an arm.

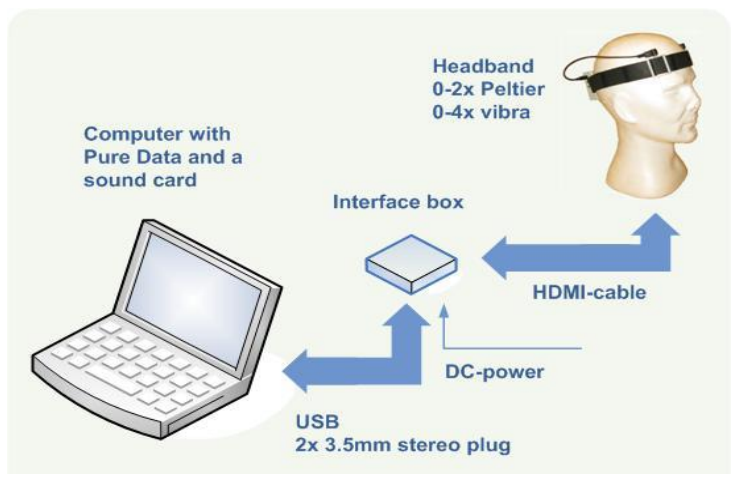

Figure 1: The prototype system for thermal and vibrotactile feedback

The band's main module was able to receive digital and analog feedback signals from the computer, process or amplify them and feed them to the actuators. The thermal actuator was based on the Peltier effect in which an electric current between two metal plates causes a temperature difference between them, i.e. one of them heats up and the other one cools down. The Peltier actuator module contained one Multicomp PF-031-10-25 thermoelectric module, attached to an aluminium heatsink with double-sided heat-conducting tape. The aluminium heatsink helped to keep the temperature of the other plate of the Peltier element stable. The vibrating motor actuator module contained one Minebea LVM8 linear vibration motor that is optimally driven with 140$160 \mathrm{~Hz}$ square wave.

In the user study vibration was demonstrated with four vibrating motor actuators attached to the band. We used three different demonstration stimuli:

1) All four actuators vibrate at the same time for 3 seconds.
2) All four actuators vibrate sequentially for 2 seconds one after the other.

3) A sequence in which the vibration is "hopping" very quickly from actuator to actuator.

Thermal feedback was demonstrated with two Peltier actuators attached to the band. The demonstration was done using four single 5 second stimuli in four different temperatures. The exact temperatures were not measured, but they were relative to the surrounding temperature (the air or the skin in contact) as follows:

- Hot: $+20^{\circ} \mathrm{C}$ (relative to the surrounding temperature)

- Warm: $+10^{\circ} \mathrm{C}$

- Cool: $-7^{\circ} \mathrm{C}$

- Cold: $-14^{\circ} \mathrm{C}$

In addition to static thermal stimuli, temperature changes were also demonstrated by changing fast from one temperature stimulus to the other while the user kept in contact with the actuator. The differences between the temperatures were made clearly distinguishable from each other for demonstration purposes as the goal was not to study the interaction or detection of thermal feedback, but to use it as stimulus for ideation.

\subsection{Participants and methodology}

\subsubsection{Participants}

In four focus groups there were 15 participants in total (3-4 per session), of which seven were female and eight were male. The ages ranged from 21 to 60 years (average: 32.8, median: 27). They were recruited using the university's electronic notice board and a mailing list for recruiting user study participants. Most (12) were university students or graduates and the rest had vocational education. Their fields of education varied and included, for example, economics, computer science, materials engineering and forestry. They reported having used a variety of touch-screen devices, for example mobile phones, computers, gaming devices and mp3-players. Vibration was a familiar feedback type for all, but none had used devices that give (intentional) thermal feedback to the user.

\subsubsection{Methods}

In this study we used two common methods of human-computer interaction $(\mathrm{HCl})$ research. The study was conducted using focus groups, which is a qualitative research method for gathering participants' opinions, expectations and ideas on a given discussion topic and where the interaction between the participants acts as an important part of the data (Morgan, 2002). These qualities of focus groups make them excellent for facilitating discussion and for generating and evaluating ideas. 
In addition, we used scenarios, which are stories about people and their activities with technology (Carroll, 2000). The benefits of scenarios include that they are concrete, flexible, and they evoke reflection and promote communication about their content (Carroll, 2000). Scenarios are shown to be a useful way to concretely embody a view of users' actual and future activities (Nigay et al. 2002). In our approach the participants of focus groups themselves created use scenarios of communication situations in which they would like to use haptics.

\subsubsection{Procedure}

The focus group sessions started with an introduction to the study and the related haptic concepts. Then a semi-structured interview was used to raise discussion among the participants: first, about communication in general, and then about haptics and how thermal and vibrotactile feedback could be used in new ways in existing communication devices such as mobile phones.

Next, the participants tested the prototype one by one, first by touching the actuators with their fingers and then on any other body parts, if they wanted. All of them tested both feedback types with their fingers, most also on their heads, some on their wrists or the back of their hands. The discussion continued based on the prototype: ideas for using the device, and benefits and problems of using it in communication.

Then, based on the discussion and prototype testing, the participants created use scenarios for interpersonal haptic communication in pairs or groups of three people. Ideas concerning both the existing technologies and possible future applications were welcomed. The scenarios were briefly discussed within the group. Finally, the participants' perceptions of using vibrotactile and thermal feedback in communication were studied with a questionnaire with eight statements on a 7point Likert scale with anchors "I strongly disagree" and "I strongly agree".

Research data was collected by audio recording the sessions and documenting the created scenarios and the questionnaire. In the analysis phase the scenarios were studied and the audio recordings of the discussions were transcribed and both were analyzed qualitatively. The questionnaire data was analyzed quantitatively.

\section{USER STUDY OF SQUEEZE FEEDBACK}

The goal of the second study that consisted of six pair interviews was to explore users' ideas and expectations about potential uses of squeeze feedback in interpersonal communication, with the help of a squeeze-band prototype. Similarly as in the first study, we wanted to study people's perceptions of how, in what kind of situations, and for what communication purposes squeeze feedback could be used.

\subsection{The squeezing wristband prototype}

The squeezing wristband prototype (see Figure 2) consisted of two shape memory alloy (SMA) MigaOne actuators, a control electronics board and a non-elastic adjustable wristband (test unit with Suunto Core Flat Strap). The prototype could be operated with a push-button or with a computer via a USB-port. The prototype also provided data on the amount of squeeze via capacitive sensing. Speed and the percentage of actuation were controlled via serial commands.

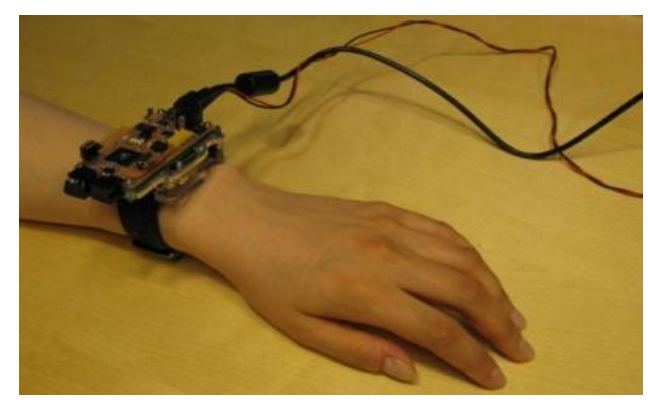

Figure 2: The squeeze-band prototype

The chosen actuator can be used in applications that call for linear movement. Two actuators were used because the actuator has a slide on only one side of it, and uniform movement required dualsided actuation. SMA actuator has consecutive wires which contract as they heat up when an electrical current flows through them. When the wires cool down the actuator retracts to its resting position.

Five different squeeze stimuli were used to demonstrate the device and squeezes around the wrist. The five stimuli included two different single squeezes and three different short sequences with varying speed and length of travel. These were not based on any specific use cases as their purpose was only to demonstrate this novel interaction technique for the users. The stimuli were sent to the band from a laptop computer. The maximum speed of the squeeze actuation was 1 second for full actuation and the maximum travel of the squeeze was 9 millimetres.

\subsection{Participants and methodology}

\subsubsection{Participants}

We conducted six pair interviews with 12 participants in total. Their recruitment was done similarly as in the first study. The pairs knew each other beforehand: four of them were couples 
(dating or living together) and two were friend pairs. Their ages varied from 21 to 26 (average: 23.3, median: 23) and there were seven females and five males. One pair consisted of two girls (friends) but all others were mixed pairs. Nine of them were studying technology (different fields of engineering) in a university and the rest had polytechnic education, for example in nursing and tourism industry. All but one of them reported having used touch screen devices (e.g. mobile phone), nine had used wearable technology (e.g. heart rate monitor, pedometer) and all had used devices that give feedback through the sense of touch, but mostly only vibrotactile feedback. None of them had used devices that give squeeze feedback to the user.

\subsubsection{Methods and procedure}

The interview sessions began with an introduction to the study. Then the interview started with background questions about the participants' earlier experiences and expectations of using related technology (i.e. touch-based, wearable, squeezing) and their habits of using the sense of touch in traditional face-to-face communication. Next, the participants tested the squeeze prototype one after the other around their wrist and forearm. Then the interview continued with focus now on the device: 1) how the squeezes felt, 2) ideas for the design of the device, and 3 ) how it could be used in communication.

The interview was followed by a small task for identifying the best potential use cases for squeeze feedback. The approach was changed from the free ideation that was used in the first study to selecting the best use cases from a precreated list to better keep the ideation on the main focus: interpersonal communication. The pairs were presented with 23 notes with potential use cases for haptic feedback in communication, for example "communicating joy," "comforting the other" and "communicating a route." The notes included both emotion-related and pragmatic communication purposes. The set of 23 use cases was precreated by us based on publications about communicating emotions with haptics (Bailenson et al. 2007; Heikkinen et al. 2009b) as well as mobile communication and text messaging (Battestini et al. 2010). These publications present several potential uses for mobile and haptic communication, from which only the most clearly inapplicable ones for our study were dismissed and the rest were combined and partly rephrased to form our 23 use case notes.

The pairs were asked to first select from the notes 10 use cases that they thought were best for using the squeeze-band device and in which they would like to use the device. Then they were asked to narrow down their selection and choose three best use cases from those 10. At this point they were also given the chance to replace some of the three best use cases with their own ideas, if they felt they had better ideas in their mind. Finally they were asked to ideate and explain in detail on a concrete level how the squeeze device would be used in the three best use cases they had finally selected.

Finally the participants filled out a questionnaire about their perceptions of using squeeze feedback in communication, which consisted of nine statements on a 5-point Likert scale (later transformed to 7-point for comparison of results) with anchors "I strongly disagree" and "I strongly agree". Eight of the statements were the same as in the questionnaire in the first study.

Research data was collected by audio recording the sessions, and by documenting the questionnaire and task results. The task results (i.e. the selected use cases and their descriptions) were analyzed qualitatively. The interview recordings were transcribed and analyzed qualitatively. The questionnaire data was analyzed quantitatively.

\section{RESULTS}

The results of the two studies are presented combined in the following subsections: the experiences with the different feedback types; the ideas for potential use scenarios for haptic feedback; expectations of use; and finally, the results of the questionnaire.

\subsection{Experiences with the three haptic feedback types}

\subsubsection{Vibrotactile feedback}

The participants experienced that the most intensive vibrations sounded loud (especially on the head) and felt unpleasant, but weak vibration was not felt at all. Different kinds of sequences were easy to distinguish from each other and it was suggested they could be used to convey different kinds of messages. The fast and "hopping" sequence was experienced as the most fun. One participant said the vibrations felt fun on the head, but another one said they were unpleasant and should not be used on the head at all.

\subsubsection{Thermal feedback}

The hottest thermal stimulus was described as "unpleasant" and "too hot" and the coldest was "freezing, hurting and stinging." Overall, cold was easier to notice than warmth and experienced as better for communicating things than hot feedback. The cool and warm temperatures between the extreme ends were experienced as more pleasant than the extremes but not as easy to feel. In addition, the participants noted that changes are easier to notice than static temperatures. They also felt that complicated messages should not be 
conveyed with temperatures, since thermal stimuli are sensed slowly and the skin adapts to the temperatures after a while, after which sensing becomes more difficult.

\subsubsection{Squeeze feedback}

All of the pairs said at some point that the demonstrated squeezes were surprisingly weak, maybe even too weak, and that they could be a lot stronger, provided that they don't last very long: "they can be felt when you focus on them, but what happens if you are, for example, walking?" Faster squeezes were experienced as better than slow squeezes, because they are more noticeable, and for the same reason sequential squeezes were preferred over single ones. Some participants associated the squeezes with the feeling of someone grabbing their wrist (in a positive sense). One participant described them as very pleasant and even massage-like. None of the participants described the squeezes as unpleasant in any way.

\subsection{Use scenarios for haptic communication}

\subsubsection{Vibrotactile and thermal feedback}

In the first study the participants created collaboratively seven use scenarios for vibrotactile and thermal feedback in communication. However, three of them did not include interpersonal communication at all and therefore are discarded from these results. Summaries of the four remaining scenarios are presented in Table 1.

Three of the scenarios include only thermal feedback and one has a combination of thermal and vibrotactile feedback (scenario 1). The mobile phone is the main technology in three scenarios and one involves using an instant messaging application (scenario 3). Two themes can be identified from the scenarios: a) communicating emotions or moods (scenarios 1, 3 and 4) and b) using thermal feedback to notify something to someone (scenario 2). The participants also discussed the scenarios briefly and concluded that attaching thermal feedback to, for example, text messages would be a great way to express feelings and moods, even better than smileys.

\subsubsection{Squeeze feedback}

The results of the task in the second study are presented in Table 2 on the next page: nine use cases from our precreated list, which were selected by one or more of the pairs to be among the three best uses for the squeeze-band device. Summaries of the participants' ideas of how the device could be used in those use cases are also presented. None of the pairs used the opportunity to add their own ideas to their lists of three best use cases.
Table 1: User-created scenarios for vibrotactile and thermal feedback

\begin{tabular}{|l|l|}
\hline $\begin{array}{l}\text { Scenario } \\
\text { name }\end{array}$ & Summary of the scenario \\
\hline mind! Never- & $\begin{array}{l}\text { Two friends have agreed to meet but one } \\
\text { of them postpones the meeting } \\
\text { repeatedly. The other gets angry and } \\
\text { sends a hot and vibrating message from } \\
\text { her phone (phones have haptic } \\
\text { actuators meaning "Nevermind!" But } \\
\text { without a verbal message, correct } \\
\text { interpretation can be difficult. }\end{array}$ \\
\hline $\begin{array}{l}\text { 2. Pick up } \\
\text { from home }\end{array}$ & $\begin{array}{l}\text { Mother is picking her daughter up from } \\
\text { her flat, but because of traffic, she } \\
\text { doesn't know when she will be there. } \\
\text { They agree on the phone that the mother } \\
\text { will send a thermal message when she is } \\
\text { at the house, so the daughter knows } \\
\text { when to come outside to the road side. }\end{array}$ \\
\hline $\begin{array}{l}\text { 3. Emotio- } \\
\text { nal com- } \\
\text { munication } \\
\text { in instant } \\
\text { messaging }\end{array}$ & $\begin{array}{l}\text { A daughter is in exchange abroad and } \\
\text { has an IM conversation with her mother. } \\
\text { They start by saying hello with warm } \\
\text { thermal feedback to show intimacy. Then } \\
\text { the daughter sends a cooler feedback to } \\
\text { indicate that she has bad news: she has } \\
\text { spent all her money and asks for more. } \\
\text { The mother replies with a cold feedback } \\
\text { to tell that she is angry. Finally they come } \\
\text { to terms about sending the money and } \\
\text { send each other warm feedback to show } \\
\text { that they are happy with the decision. }\end{array}$ \\
\hline $\begin{array}{l}\text { 4. Trans- } \\
\text { ferring } \\
\text { emotions } \\
\text { and moods }\end{array}$ & $\begin{array}{l}\text { A boy wants to express his feelings to a } \\
\text { girl by sending her a warm text message } \\
\text { saying "Hi!" He adds a "thermal } \\
\text { attachment" for the message from his } \\
\text { phone's menu to express his warm } \\
\text { feelings. When the girl reads the } \\
\text { message, she will feel the warmth. }\end{array}$ \\
\hline
\end{tabular}

In total, four pragmatic (use cases 1, 2, 3 and 8) and five emotion-related (4, 5, 6, 7 and 9) use cases were selected. Pragmatic use cases were more popular, according to the number of times they were selected (see Table 2).

Also during the interviews some ideas were expressed. For instance, if the device was connected to a mobile phone, the user could squeeze the phone during a call and the band would squeeze the receiver's wrist. It could also replace the vibration alarm - a squeeze is easier to feel and more silent. Squeezing could be used to convey simple messages, for getting someone's attention, as an aid for people with sensory disabilities and for communicating emotions. One pair suggested that if heat were added to the squeeze, it could feel like the touch of another person. At the end of the interviews, all interviewees said they would like to use or at least test the device in real use situations. 
Table 2: The best use cases for the squeeze-band and participants' ideas of the usage in each case

\begin{tabular}{|l|l|}
\hline Use case & Summary of usage ideas \\
\hline $\begin{array}{l}\text { 1. Notification/ } \\
\text { reminder } \\
\text { (selected by 6 } \\
\text { pairs as one of } \\
\text { the best three } \\
\text { use cases) }\end{array}$ & $\begin{array}{l}\text { The device could be connected to } \\
\text { applications in mobile phone or } \\
\text { computer. Different squeezes for } \\
\text { different notifications (e.g. fast and } \\
\text { repeating for urgent issues) and for } \\
\text { separating messages from different } \\
\text { sources. Could also be used with } \\
\text { sports equipment to notify things. }\end{array}$ \\
\hline $\begin{array}{l}\text { 2. Communica- } \\
\text { ting a route or } \\
\text { location } \\
\text { (3 pairs) }\end{array}$ & $\begin{array}{l}\text { The device could be connected to } \\
\text { mobile phone or navigator, or have } \\
\text { integrated GPS. Squeezes tell the } \\
\text { user where to go: e.g. when to turn } \\
\text { or how close the target is. }\end{array}$ \\
\hline $\begin{array}{l}\text { 3. Asking a } \\
\text { question } \\
\text { (2 pairs) }\end{array}$ & $\begin{array}{l}\text { Short and simple predefined } \\
\text { questions, meaning defined by the } \\
\text { number and intensity of squeezes. }\end{array}$ \\
\hline $\begin{array}{l}\text { 4. Communica- } \\
\text { ting that you } \\
\text { care about the } \\
\text { other } \\
\text { (2 pairs) }\end{array}$ & $\begin{array}{l}\text { For sending positive feelings to } \\
\text { close ones. Squeezes are e.g. } \\
\text { slow, gentle, massaging or } \\
\text { cheering. The device would be } \\
\text { used alone or connected to a } \\
\text { phone. }\end{array}$ \\
\hline $\begin{array}{l}\text { 5. Comforting } \\
\text { the other (1 pair) }\end{array}$ & $\begin{array}{l}\text { Similar to showing that you care. } \\
\text { Calm but strong squeezes. }\end{array}$ \\
\hline $\begin{array}{l}\text { 6. Communica- } \\
\text { ting excitement } \\
\text { (1 pair) }\end{array}$ & $\begin{array}{l}\text { Fast and strong squeezes to } \\
\text { indicate that you are currently } \\
\text { seeing or experiencing something } \\
\text { exciting. }\end{array}$ \\
\hline $\begin{array}{l}\text { 7. Communica- } \\
\text { ting empathy } \\
\text { (1 pair) }\end{array}$ & $\begin{array}{l}\text { Squeezes could augment speech } \\
\text { (easier to know the meaning). The } \\
\text { nature of the squeezes depends on } \\
\text { the situation being empathized. }\end{array}$ \\
\hline $\begin{array}{l}\text { 8. Answering a } \\
\text { question } \\
\text { (1 pair) }\end{array}$ & $\begin{array}{l}\text { Squeeze to answer to simple } \\
\text { questions (yes/no) or tell the sender } \\
\text { that you received their message. }\end{array}$ \\
\hline $\begin{array}{l}\text { 9. Having fun } \\
\text { (1 pair) }\end{array}$ & $\begin{array}{l}\text { Games or similar activity, e.g. the } \\
\text { squeezes increase when you are } \\
\text { getting closer to a specific place, } \\
\text { person or object. }\end{array}$ \\
\hline
\end{tabular}

\subsection{Expectations of haptic communication}

\subsubsection{Most important benefits}

The participants mentioned in both studies that one of the most important benefits of haptic feedback is that it is a new channel for conveying information via technology and brings a whole new dimension to communication. The three haptic feedback types were seen as allowing richer and more efficient communication than traditional methods.

Furthermore, the participants felt that haptic feedback offers an immediate, intuitive and easy way to express emotions, as touch is an important element also in traditional face-to-face communication, especially in emotional contexts. One of the best benefits of thermal and squeezing feedback, according to the study participants, is their intimacy. As opposed to vibration, which usually causes a low sound, they are completely silent and unnoticeable by outsiders. All three feedback types were seen as immediate ways of communicating as the messages come directly to the user's skin.

\subsubsection{Potential problems}

In both studies the participants felt that correct interpretation of messages can be difficult - what does a specific vibration, thermal cue, or squeeze mean? Furthermore, it is possible to miss something from the start of a feedback sequence and thus not get the whole message. Also, there can be subjective differences between users' sense of touch and their ability to sense the stimuli some feel them weaker and some stronger.

On the other hand, there might be problems in feeling the feedback due to for example user's movements, badly attached devices, and other disturbances diminishing the user's ability to sense the stimuli, such as the temperature of the surroundings in the case of thermal stimuli. It was suggested that there should be a way to guarantee that messages are received, even if the user is not touching the device all the time. For example, the device could sense when it is being touched and only then give the haptic message. Some participants were worried about safety issues, such as potential skin damage, if a thermal feedback device accidentally burns the skin, or if the squeeze-band squeezes the wrist too strongly.

\subsubsection{Usability and wearability of haptic devices}

According to the participants, thermal and vibrotactile actuators could be integrated into many types of existing devices for different communication purposes. They mentioned devices such as mobile phones, computer mouse, wristwatch/computer, navigator devices, gaming devices and sports equipment. On the other hand, actuators could be sewn into clothing or hats to create wearable communication technology with the benefit of having actuators directly on the user's skin. Hands and arms were preferred over other body parts for receiving stimuli, and especially the wrist was seen as a natural body part to place a haptic device. A common requirement for all applications was that they would be wireless.

Regarding the further design of the squeeze-band, it was wished that it would look like a wristwatch, a decorative bracelet or just a simple band made of rubber, plastic, leather or soft fabric. The device could also have a small display for communicating additional information, for example the sender of a haptic message. The participants noted that optionally the squeezing mechanics could be integrated into existing devices, such as a wristwatch or heart-rate monitor. All participants felt that the wrist is the best location for the squeezeband, but also forearm, upper arm, ankle and finger (e.g. a ring) were listed as possible locations for 
wearing it. In all cases the device should be small, light, comfortable on the skin and wireless.

\subsection{Questionnaire results}

A questionnaire with eight statements (scale: $1=1$ strongly disagree, $7=$ I strongly agree) was used in both studies to explore the participants' perceptions of using vibrotactile, thermal and squeeze feedback in communication. The means of the evaluations are combined in Figure 3, with standard deviation.

In pairwise comparison using the Mann-Whitney Utest, significant differences were found between vibrotactile and thermal feedback in response to the statement "I would like to use $X$ feedback to communicate my emotions" (means: 3.5 and 5.1, $\mathrm{p}<0.05$ ) and between vibrotactile and squeeze feedback in response to the statement "I would like to use $X$ feedback to communicate utility information" (means: 5.3 and 6.3, p<0.05). There were no significant differences between thermal and squeeze feedback. In addition to the eight statements, the squeeze study included a ninth statement: "Using squeeze feedback would increase the feeling of closeness between me and my communication partner," with mean evaluation of 4.5 (sd: 2.34).

The questionnaire results indicate that all of the feedback types would be used to communicate utility information rather than emotions. Squeeze feedback was evaluated as the best of the three for communicating utility information and thermal feedback was the best for communicating feelings, with only a marginal difference in its potential to communicate utility information.

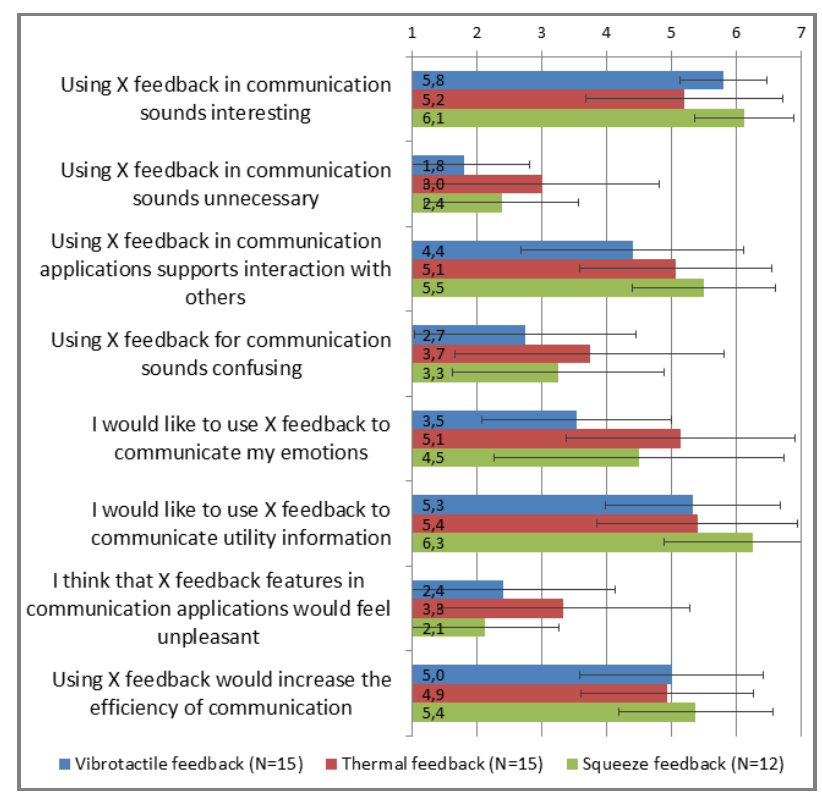

Figure 3: Perceptions of using haptic feedback in communication

\section{DISCUSSION AND CONCLUSIONS}

In this paper we have presented two studies on haptic feedback, focusing on user perceptions of using vibrotactile, thermal and squeezing feedback in interpersonal communication. We used early prototypes of the haptic devices as stimulus materials in the two user studies, with altogether 27 participants.

As expected, vibration was a familiar feedback type for the participants from their mobile phones, but they suggested using it in new ways, for example in sequences to convey different kinds of messages. Regarding thermal stimuli, the user perceptions suggest that it might be challenging to determine which temperatures should be used for feedback, so that they are not too hot or cold, but can still be sensed. Overall, cold stimuli were experienced as more pleasant than warm and changes were easier to notice than static temperatures. Furthermore, the participants would prefer to receive thermal stimuli to their hand-area, through a mobile phone, a wristband-like device or wearable technology in the form of clothing. These user perceptions concerning the detection of thermal stimuli are in line with the results obtained in earlier interaction studies, for example, by Wilson et al. (2011).

The squeeze-band prototype evoked slightly more positive comments than the prototype with thermal and vibrating actuators. The squeezes were experienced as very pleasant and even stronger squeezes were hoped for. Similarly, as with vibration and thermal feedback, sequences were seen as a more usable way to give feedback than single squeezes. The wrist was perceived as the most natural location to wear a squeezing device. In both studies most of the concepts in the ideated scenarios were mobile in one way or another, which reflects the characteristic of haptic interaction that the devices are used literally on the skin and thus they are usually always with the user.

The participants' saw the biggest potential problems of haptics to be the same for all three feedback types. First of all, there can be problems in sensing the feedback for various reasons, such as poor contact between the skin and actuators. Second, even if haptic messages are received properly, their correct interpretation can be challenging. However, this issue is already being tackled by researchers focusing on the creation and interpretation of haptic messages, such as Rantala et al. (2011) and Heikkinen et al. (2011). On the positive side, haptic feedback in general was described as bringing a new and rich dimension to communication, and being efficient, intuitive, intimate, and immediate. 
It seems that people perceive haptic feedback as a positive aspect of their future communication devices. The newer feedback types - thermal and squeeze - were often connected to pragmatic functions similar to those for which vibration is nowadays being used, for example notifications and alarms. The participants also found use for haptics in conveying simple messages, such as yes-no answers. Using technology to communicate feelings or moods was generally approved, but some participants still preferred to communicate emotions without technology. However, haptics does have its use cases also in emotional contexts, for example in showing that you care about a friend who is too far for face-to-face caring. Some participants associated the gentle but firm squeeze of the squeeze-band around their wrist to another person grabbing their hand - a sensation that could be further enhanced by adding warm thermal feedback to it. Overall, haptics was seen as a type of interaction that would be mostly used with close people, such as friends and family, because of its intimate nature.

The questionnaire results confirmed the participants' generally positive perceptions of haptic feedback and highlighted some of the differences between the three feedback types, for example, that squeezes were experienced best for utility purposes and thermal feedback for communicating emotional messages. However, it should be noted that the comparisons we made between the feedback types were based on the results of two separate studies, which had different participants and were not identical, even though essentially very similar. Still, the quantitative comparisons are in line with the qualitative user feedback and this method triangulation increases the validity of the results.

Regarding the methodology, the presented studies confirm the finding of Mueller et al. (2005) that even early technology prototypes are valuable tools in user studies. Our prototypes succeeded in their role - demonstrating haptic feedback and evoking conversation among the potential future users of these technologies - both in focus groups as well as in pair interviews. This approach is in line with the experience prototyping approach presented by Buchenau and Fulton Suri (2000) concerning exploration of ideas and experiences together with the potential users. Another related method are technology probes (Hutchinson et al. 2003) which can be used as inspirations for users and researchers to think and ideate about new technologies. The prototypes we used provided the participants with something tangible that they could base their comments on, instead of merely imagining what haptic interaction might feel like. And still, the prototypes were not too advanced, which left the participants with enough freedom to develop their own ideas. We also designed such demonstration stimuli that they would not be associated to any specific use cases. We argue for the value of such tangible prototypes especially for haptics research, where the experiences are hard to imagine or evaluate using low-fidelity prototypes.

Although some of the participants' scenarios and use case descriptions were left somewhat shallow, all of the ideas are still valuable and include important information that can be used in future studies. It requires further design effort to conclude how the devices actually would be used in some situations, for example how to ask a question with mere squeezes.

The main contribution of this research is twofold: first of all, the results include insights about potential users' expectations, experiences and ideas for using haptic feedback in different communication scenarios and their overall perceptions of the topic. Second, these studies illustrate the value of using early prototypes for example as a part of focus groups in the early stages of research processes to stimulate idea generation and explore experiences. In our future work we are planning to develop more advanced prototypes, focusing on hand-area haptic feedback with combinations of different types of haptic interaction. We also intend to continue user studies both in laboratory and real contexts to gain broad picture of user experience of haptic feedback in communication.

\section{ACKNOWLEDGEMENTS}

We would like to thank Jussi Rantala and Jukka Raisamo for their contribution in implementing the prototypes. The research was part of the project Human Emotional Interaction, funded by Nokia and the Finnish Funding Agency for Technology and Innovation (TEKES).

\section{REFERENCES}

Bailenson, J.N., Yee, N., Brave, S., Merget, D. and Koslow, D. (2007) Virtual interpersonal touch: Expressing and recognizing emotions through haptic devices. Hum.-Comput. Interact. 22, 3, August 2007, 325-353.

Battestini, A., Setlur, V. and Sohn, T. (2010) A large scale study of text-messaging use. In Proceedings of MobileHCl '10, pp. 229-238. ACM, New York, NY, USA.

Baumann, M.A., MacLean, K.E., Hazelton, T.W. and McKay, A. (2010) Emulating human attentiongetting practices with wearable haptics. In Haptics Symposium, 2010 IEEE, 25-26 March, pp.149-156. 
Buchenau, M. and Fulton Suri, J. (2000) Experience prototyping. In Proceedings of DIS'00, pp. 424-433. ACM Press, New York, NY, USA.

Carroll, J. M. (2000). Five reasons for scenariobased design. Interacting with Computers, 13, 4360.

Chang, A., O'Modhrain, S., Jacob, R., Gunther, E. and Hiroshi, I. (2002) ComTouch: Design of a vibrotactile communication device. In Proceedings of DIS'02, pp. 312-320. ACM Press, NY.

Gooch, D. and Watts, L. (2010) Communicating social presence through thermal hugs. In Proceedings of First Workshop on Social Interaction in Spatially Separated Environments, SISSI 2010.

Haans, A. and ljsselstein, W. (2006) Mediated social touch: A review of current research and future directions. Virtual Reality, vol. 9, no. 2, 149159.

Hassenzahl, M. (2004) The thing and I: Understanding the relationship between user and product. In Blythe, M. A., Overbeeke, K., Monk, A. F. and Wright, P. C. (eds), Funology: From usability to enjoyment, pp. 31-42. Kluwer Academic Publishers, Norwell, MA.

Heikkinen, J., Olsson, T. and Väänänen-VainioMattila, K. (2009a) Expectations for user experience in haptic communication with mobile devices. In Proceedings of MobileHCl '09, article 28, 10 pages. ACM, New York, NY, USA.

Heikkinen, J., Rantala, J., Olsson, T., Raisamo, R., Lylykangas, J., Raisamo, J., Surakka, V. and Ahmaniemi, T. (2009b) Enhancing personal communication with spatial haptics: Two scenariobased experiments on gestural interaction. J. Vis. Lang. Comp. 20, 5, October 2009, 287-304.

Heikkinen, J., Rantala, J., Olsson, T., Raisamo, R. and Surakka, V. (2011) Exploring the effects of cumulative contextual cues on interpreting vibrotactile messages. In Proceedings of MobileHCl '11, pp.1-10. ACM, New York, NY, USA.

Hutchinson, H., Mackay, W., Westerlund, B., Bederson, B., Druin, A., Plaisant,, C., BeaudouinLafon, M., Conversy, C., Evans, E., Hansen, Roussel, H., Eiderbäck, B., Lindqvist, S. and Sundblad, Y. (2003) Technology probes: Inspiring design for and with families. In Proceedings of $\mathrm{CHI}$ 2003, pp. 17-24. ACM, New York, NY, USA.

Lee, W. and Lim Y-K. (2010) Thermo-message: Exploring the potential of heat as a modality of peripheral expression. In Proceedings of $\mathrm{CHI} E A$ 2010, pp. 4231-4236. ACM, New York, NY, USA.

Morgan, D.L. (2002) Focus groups as qualitative research. Second ed., Sage Publications Inc., California.

Mueller, F., Vetere, F., Gibbs, M., Kjeldskov, J., Pedell, S. and Howard, S. (2005) Hug over a distance. In Proceedings of CHI EA '05, pp. 16731676. ACM, New York, NY, USA.

Nigay, L., Salembier, P., Marchand, T., Renevier, P. and Pasqualetti, L. (2002) Mobile and collaborative augmented reality: A scenario based design approach. In Proceedings of the 4th International Symposium on Mobile HumanComputer Interaction (Pisa, 18-20 September 2002), LNCS, Vol. 2411, pp. 241-255. SpringerVerlag, Berlin.

Rantala, J., Raisamo, R., Lylykangas, J., Ahmaniemi, T., Raisamo, J., Rantala, J., Mäkelä, K., Salminen, K. and Surakka, V. (2011) The role of gesture types and spatial feedback in haptic communication. Haptics, IEEE Transactions on , vol.4, no.4, July-Aug, 295-306.

Salminen, K., Surakka, V., Raisamo, J., Lylykangas, J., Pystynen, J., Raisamo, R., Mäkelä, K., and Ahmaniemi, T. (2011) Emotional responses to thermal stimuli. In Proceedings of ICMI'11, pp. 193-196. ACM, New York, NY, USA.

Teh, J., Cheok, A., Peiris, R., Choi, Y., Thuong, V. and Lai, S. (2008) Huggy Pajama: A mobile parent and child hugging communication system. In Proceedings of IDC '08, pp. 250-257. ACM, New York, NY, USA.

Vaucelle, C., Bonanni, L. and Ishii, H. (2009) Design of haptic interfaces for therapy. In Proceedings of $\mathrm{CHI}$ '09, pp. 467-470. ACM, New York, NY, USA.

Wang, R. and Quek, F. (2010) Touch \& Talk: Contextualizing remote touch for affective interaction. In Proceedings of TEI 2010, pp. 13-20. ACM, New York, NY, USA.

Wettach, R., Behrens, C., Danielsson, A. and Ness, T. (2007) A thermal information display for mobile applications. In Proceedings MobileHCl '07, pp. 182-185. ACM, New York, NY, USA.

Wilson, G., Halvey, M., Brewster, S.A. and Hughes, S.A. (2011) Some like it hot: Thermal feedback for mobile devices. In Proceedings of $\mathrm{CHI}$ '11, pp. 2555-2564. ACM, New York, NY, USA. 